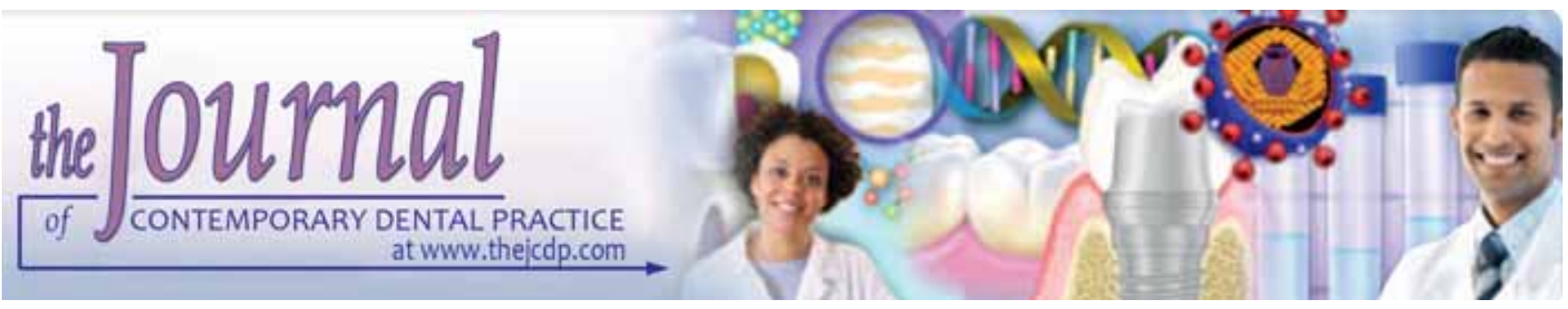

\title{
Assessment of Interobserver Variability in Mitotic Figure Counting in Different Histological Grades of Oral Squamous Cell Carcinoma
}

\author{
K Shailaja Yadav, Sudhir Gonuguntla, Kranti Kiran Reddy Ealla, Surekha Reddy Velidandla, CR Charan Reddy \\ MD Prasanna, Sanjay Reddy Bommu
}

\begin{abstract}
Mitotic counting is often used for classification, grading and prognosis of tumors. The count usually stands as a decision point for treatment as well. The easiest way of counting the number of mitoses is done by screening routine $H \& E$ stained slides. However, for proper mitotic counting, certain strict protocols should be taken into consideration. This study on 30 cases of different grades of oral squamous cell carcinoma was undertaken to determine the interobserver variations in two different groups: Group1 (A1, A2), who were given certain criteria to be followed during the counting of the mitotic figures and group 2 investigators (B1, B2) who were unaware of such criteria. The paired t-test gives a correlation of 0.988 and a significant difference of 0.000 between the two investigators in group 1. The correlation was 0.650 with a significant difference of 0.058 between two investigators in-group 2, indicating that group 1 observers exhibit good interobserver agreement. The results emphasize that following of strict protocols are of great help in determining the accuracy of mitotic counting.
\end{abstract}

Keywords: Mitotic figures, Mitotic counting, Squamous cell carcinoma.

How to cite this article: Yadav KS, Gonuguntla $S$, E alla KKR, Velidandla SR, Reddy CRC, Prasanna MD, Bommu SR. Assessment of Interobserver Variability in Mitotic Figure Counting in Different Histological Grades of Oral Squamous Cell Carcinoma. J Contemp Dent Pract 2012;13(3):339-344.

\section{Source of support: Nil}

Conflict of interest: None declared

\section{INTRODUCTION}

There is an increasing awareness that proliferation is one of the most important features characterizing the malignant phenotype. In general, a high proliferative rate is found in malignant tumors and rarely in benign tumors and inflammatory conditions. ${ }^{1}$ There are several quite sophisticated ways of assessing the proliferative activity of tumors, such as:
1. Counting of cells with labelled DNA precursors (thymidine, uridine).

2. Extrapolation of the percentage of S-phase cells from DNA flow cytometry histograms.

3. Nuclear morphology.

4. Nucleolar organizing regions.

5. Nuclear antigens (PCNA, Ki 67, BK 19.9, Bromodeoxyuridine, Topoisomerase II)

6. Oncogene encoded proteins (c-myc, c-erb-B-2). ${ }^{1,2}$

$Y$ et, the oldest, easiest, fastest and cheapest way of assessing proliferation is by counting the number of mitoses in routine $H \& E$ stained slides. ${ }^{1}$ The rationale for mitotic counting is that it is frequently used for classification and grading of tumors, prediction of prognosis of tumors and even advocated as a decision point for treatment.

However, for the proper use of the study on mitotic counting, certain strict protocols should be taken into consideration. ${ }^{3}$

Protocol for proper mitotic counting includes:

1. M icroscopic requirements

2. Slide quality

3. Selection of the areas for counting

4. Criteria for selection of mitotic figure

5. Counting procedure.

The mitotic count is reported by counting $10 \mathrm{HPFs}$ (High power fields). This value is divided by 10 to get the mitotic figure count.

\section{Microscopic Requirements}

The size of the field of view (1HPF) is determined by the enlargement factors of the objective, enlargement factors of the ocular, the diameter of the field of view in the ocular and the eventual additional magnifying lenses between objective and ocular lens. For the counting of mitotic figures, 
an ordinary light microscope should be used with a 10X ocular and a 40X objective, a numerical aperture of 0.75 and the field diameter of $450 \mu \mathrm{m}$.

\section{Slide Quality}

The paraffin sections on the slide should be an adequately stretched section with a standard thickness of $5 \mu \mathrm{m}$. In a section of $5 \mu \mathrm{m}$, the objective has a depth of field at around $1 \mu \mathrm{m}$. Even though mitosis is better appreciated when the depth of focus is varied, mitoses should be counted within one depth of field range.

Suboptimal stretching increasing the thickness of the section or alters the focus which enhances the chance of counting more mitoses and thereby may induce a possible bias toward a higher number of mitoses.

\section{Selection of the Areas for Counting}

1. The most cellular region of the lesion must be selected, preferably at the periphery of the tumor avoiding those regions showing necrosis, inflammation or calcification as much as possible. As the periphery of the tumor is the most proliferating part of the carcinoma, as the peripheral region is more closely related to blood vessel supply.

2. All the fields which contain an area of less than $50 \%$ of the tumor cells must be omitted out from inclusion into the counting procedure.

3. If several areas of the tumor have met these criteria, the area subjectively found to have the highest density of mitotic figures is chosen.

4. In the most cellular region, a counting area of approximately $0.5 \times 0.5 \mathrm{~cm}$ in diameter is marked. If this area does not contain $10 \mathrm{HPFs}$, two or more areas that meet the above criteria are selected. If $10 \mathrm{HPFs}$ cannot be selected, the counting is performed in the available fields and the number of mitoses then found is extrapolated to $10 \mathrm{HPF}$ s to obtain the mitotic index (M I). ${ }^{4}$

\section{Mitotic Figures}

The analysis of mitotic figures was based on the guidelines proposed by Baak and Oort (1983) in their a manual of morphometry in diagnostic pathology.

These are some of the examples of the mitotic figures (Figs 1 to 4).

Taking all the described features, certain criteria were put forward to count the mitotic figures. They include:

1. A bsence of nuclear membrane zone.

2. A bsence of clear zone in the center.

3. Presence of hairy instead of triangular or spiky projections.

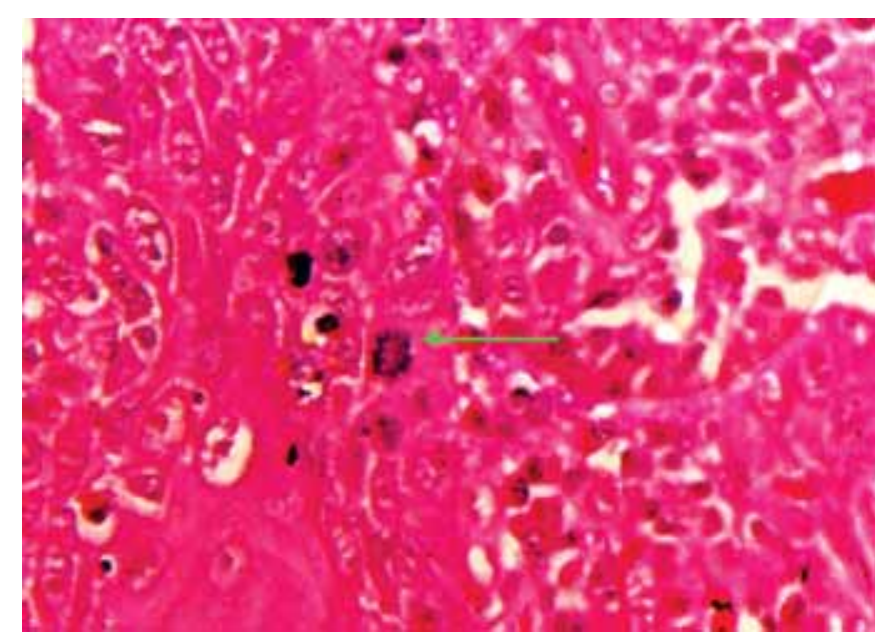

Fig. 1: Photomicrograph of a typical metaphase, with a ring of chromosomes radially oriented around an empty center in $\mathrm{H} \& \mathrm{E}$ stain, $40 x$

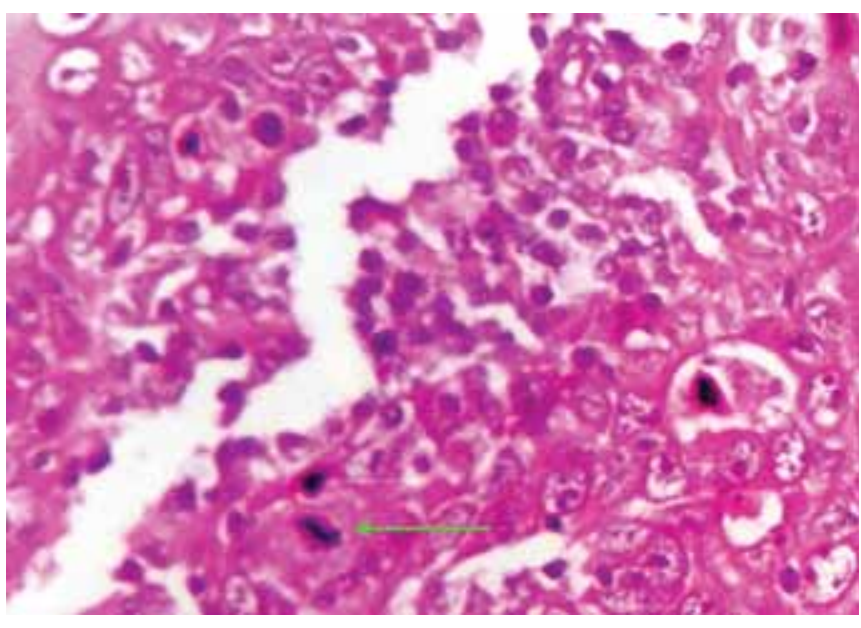

Fig. 2: Photomicrograph of typical metaphase, with dark cluster of chromosomes with hairy extensions in H\&E stain, $40 x$

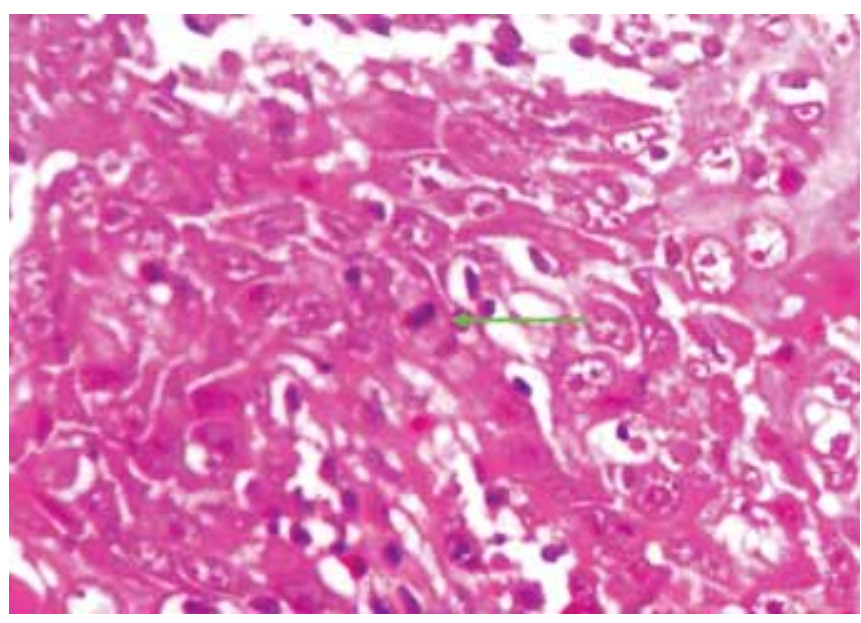

Fig. 3: Photomicrograph of typical telophase, with 2 dark clusters of chromosomes with hairy extensions lying side by side in $\mathrm{H} \& \mathrm{E}$ stain, $40 x$

4. Basophilia of surrounding cytoplasm instead of eosinophilia.

5. Structures those are not clearly identifiable, as mitotic figures should not be counted. 


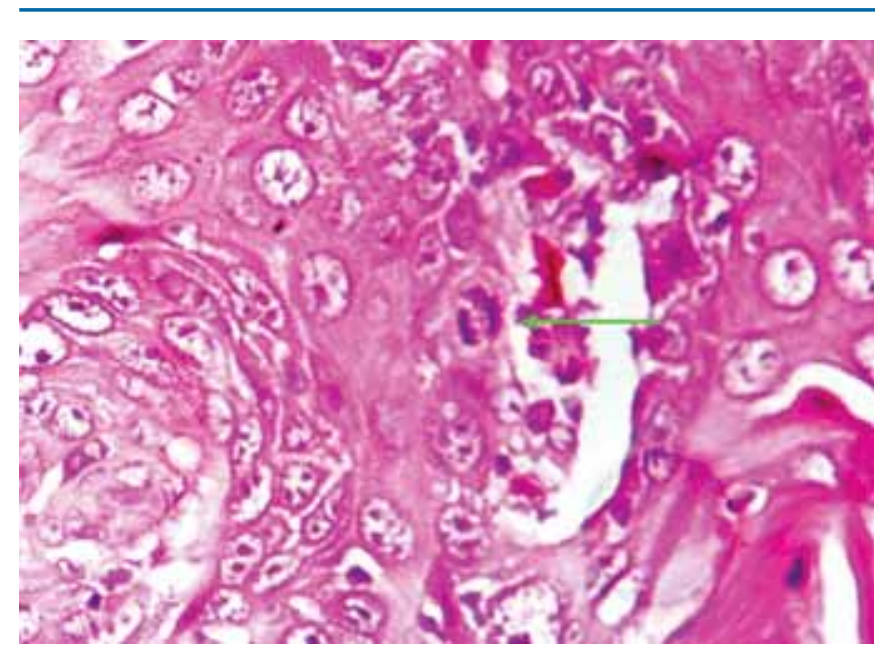

Fig. 4: Photomicrograph of a typical telophase looking like 2 close metaphase in H\&E stain, 40x

6. Two parallel clearly separate chromosomes clots are to be counted as if they are separate mitoses. $^{5}$

\section{Counting Procedure}

The high power field in which counting is to be done is bisected by a linear eyepiece micrometer. The mitotic figures intercepted by this line are counted first. Then the mitotic figures adjacent to mitotic figures in the central lines are counted carefully to avoid the inadvertent recounting of the mitotic figures. This procedure is followed for all the 10 HPFs. Finally the mitotic index is given as total number of mitotic figures per 10 HPFs.

\section{MATERIALS AND METHODS}

Thirty cases of different grades of oral squamous cell carcinoma were selected for the study. From each tumor a section was cut at $5 \mu \mathrm{m}$, prepared and after deparaffinization, routine $H \& E$ staining was done. The sections were circulated among 4 investigators who were asked to assess the mitotic figures. These investigators were divided into 2 subgroups. Group 1 investigators [A 1, A 2] were given certain criteria to be followed during the counting of the mitotic figures and group 2 [B 1, B2] was unaware of such criteria.

\section{RESULTS}

The mitotic counts of 30 cases of oral squamous cell carcinoma that were classified into well-differentiated, moderately differentiated and poorly differentiated squamous cell carcinoma respectively and estimated by 4 investigators as presented in Tables 1 to 3 and Graphs 1 to 3 .

The distribution of difference in average counts per 10 HPFs between the 2 pathologists of group 1 were as follows:

\section{For Well-differentiated Squamous Cell Carcinoma}

1 case: No difference in mitoses

4 cases: Difference of 1mitosis was observed

3 cases: Difference of 2 mitoses was observed

2 cases: Difference of 3 mitoses was observed.

\section{For Moderately differentiated Squamous Cell Carcinoma}

4 cases: No difference in mitoses

4 cases: Difference of 1 mitosis was observed 2 cases: Difference of 2 mitoses was observed.

\begin{tabular}{lrrrrrrrrrrr}
\multicolumn{10}{c}{ Table 1: Interobserver variability in 10 different cases of well-differentiated squamous cell carcinoma } \\
\hline Observer & 1 & 2 & 3 & 4 & 5 & 6 & 7 & 8 & 9 & 10 \\
\hline A1 & 13 & 14 & 17 & 15 & 12 & 11 & 15 & 14 & 12 & 13 \\
A2 & 13 & 15 & 15 & 13 & 14 & 14 & 16 & 17 & 11 & 14 \\
B1 & 15 & 18 & 9 & 6 & 17 & 11 & 10 & 9 & 16 & 7 \\
B2 & 7 & 11 & 14 & 14 & 13 & 15 & 17 & 12 & 8 & 17 \\
\hline
\end{tabular}

\begin{tabular}{lrrrrrrrrrrr}
\multicolumn{10}{c}{ Table 2: Interobserver variability } & in & \multicolumn{10}{c}{ different cases of moderately differentiated squamous cell carcinoma } \\
\hline Observer & 1 & 2 & 3 & 4 & 5 & 6 & 7 & 8 & 9 & 10 \\
\hline A1 & 16 & 18 & 21 & 17 & 19 & 17 & 16 & 19 & 18 & 22 \\
A2 & 16 & 18 & 19 & 17 & 18 & 16 & 17 & 17 & 18 & 21 \\
B1 & 18 & 23 & 26 & 21 & 22 & 20 & 11 & 12 & 27 & 26 \\
B2 & 21 & 27 & 20 & 16 & 27 & 16 & 19 & 17 & 18 & 22 \\
\hline
\end{tabular}

\begin{tabular}{lccccccrrrrr}
\multicolumn{10}{c}{ Table 3: Interobserver variability } & in 10 different cases of poorly differentiated squamous cell carcinoma \\
\hline Observer & 1 & 2 & 3 & 4 & 5 & 6 & 7 & 8 & 9 & 10 \\
\hline A1 & 31 & 25 & 28 & 26 & 29 & 32 & 27 & 27 & 28 & 30 \\
A2 & 29 & 25 & 27 & 26 & 26 & 29 & 26 & 29 & 28 & 31 \\
B1 & 20 & 33 & 21 & 28 & 22 & 26 & 34 & 21 & 23 & 26 \\
B2 & 26 & 27 & 30 & 33 & 28 & 31 & 25 & 32 & 31 & 32 \\
\hline
\end{tabular}




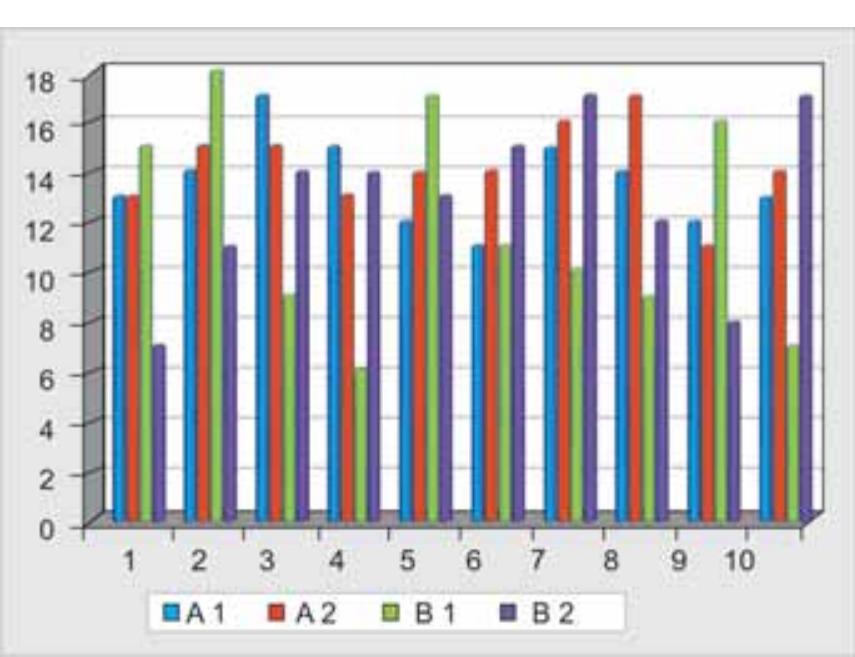

Graph 1: Interobserver variability in 10 different cases of welldifferentiated squamous cell carcinoma

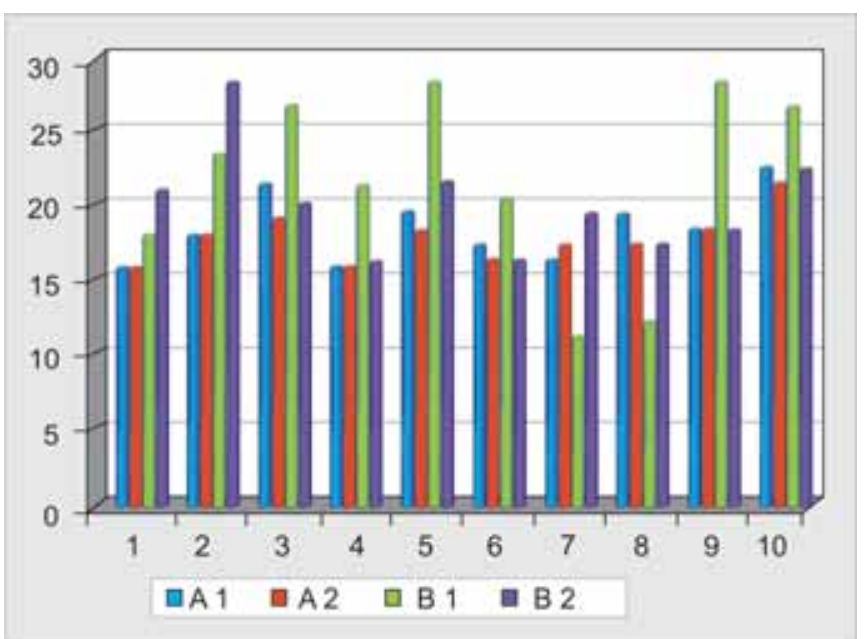

Graph 2: Interobserver variability in 10 different cases of moderately differentiated squamous cell carcinoma

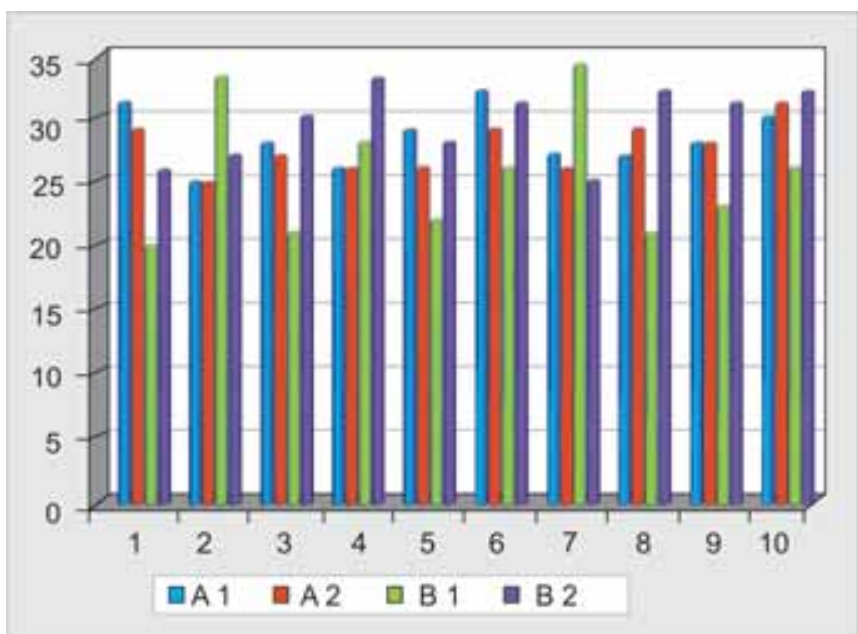

Graph 3: Interobserver variability in 10 different cases of poorly differentiated squamous cell carcinoma

For Poorly differentiated Squamous Cell Carcinoma

3 cases: No difference in mitoses

3 cases: Difference of 1 mitosis was observed
2 cases: Difference of 2 mitoses was observed

2 cases: Difference of 3 mitoses was observed.

The distribution of difference in average counts per 10 HPFs between the 2 pathologists of group 2 were as follows:

In the grade of well and moderately differentiated squamous cell carcinoma, only one case each exhibited a difference of 3 or less difference in mitoses.

In the grade of poorly differentiated squamous cell carcinoma, none of the cases exhibited a difference of 3 or less difference in mitoses.

In the present study, a difference of 0 to 3 mitoses is considered to be within the acceptable range for observer variations. Paired t-test was done to estimate the intraobserver correlation and significant difference of the observers in groups 1 and 2 respectively. The correlation between two investigators in group 1 was 0.988 with a significant difference of 0.000 . The correlation betw een two investigators in group 2 according to paired t-test was 0.650 with a significant difference of 0.058 .

From the above results, it was interpreted that the intergroup differences were higher as compared to the intragroup differences between the observers. It was also observed that the intragroup differences in ranking were higher in group 2 as compared to group 1.

Three groups were arbitrarily formed based on the mitoses and were assigned different grades of malignancy:

Grade 1: 11 to 15 mitoses/10H PFs

Grade 2: 16 to 20 mitoses/10H PFs

Grade 3: >20 mitoses/10 HPFs.

These grades were given by correlating with the histopathological diagnosis. The results were as follows: group 1 observers were correlating with the histopathological diagnosis in all the cases except for $6(54 / 60)$ whereas the group 2 observers gave the results that were not correlating in 23 out of 60 occasions.

\section{DISCUSSION}

It is an established fact that mitotic counting is an important feature for grading of malignancy. Reproducibility of the mitotic counting is paramount for the assessment of malignancy on a histologic scale. The problem of reproducibility of mitotic figures has rarely been addressed in the literature, and the few studies performed showed varied results.

The aim of this study was to evaluate the intra- and interobserver variations between the groups who have been provided with specific criteria for counting mitotic figures and those who were not provided with any criteria to do so.

The results of the present study showed that the intergroup differences were higher as compared to the intra- 
group differences between the observers clearly elucidating the fact that investigators provided with certain criteria can assess the mitoses better than investigators not provided with any criteria. It was also noted that the interobserver differences in ranking were higher in group 2 as compared to group 1. The correlation between two investigators in group 1 according to paired t-test was 0.988 with a significant difference of 0.000 . Whereas the correlation between two investigators in group 2 according to paired t-test was 0.650 with a significant difference of 0.058 . This is in agreement with the findings of $V$ an Diest et al (1992) ${ }^{4}$ who studied 2469 specimens of breast cancer and obtained reproducible mitotic counts in an investigator group comprising of pathologists of 13 laboratories. The observations in the present study are in accordance with the findings of Ellison et al $(1987)^{6}$ who in their study demonstrated that the mitotic figure as counted by two independent observers were remarkably similar with the average difference of mean count on each case being only 0.26 mitotic figures. Moreover, in $91 \%$ of patients the difference in average number of mitotic figure/ HPFs was less than 2 mitoses, thereby elaborating the fact that mitotic figures can be recognized readily with a high degree of consistency.

The findings in the present study however are contrary to the findings of Silverberg $(1976)^{7}$ and to those of Stenkvist (1979) ${ }^{8}$ who obtained extremely variable counts in mitosis counting which can be attributed to the fact that the investigators were not provided with any criteria/ guidelines before the evaluation of slides.

The altered results in group 2 can be attributed to the fact that various other structures like pyknotic nuclei, neutrophils, apoptotic cells and crushed cells that resemble a mitotic figure might have been counted in the fields that have contributed to an abnormally higher results and lower results were result of factors such as altered cell numberstromal quantity ratio, areas of inflammation, calcification and necrosis and observer restricted to count single phase of mitosis, such as metaphase or anaphase.

In the present study, the ranking and grading system were based on the protocol of D onhuijsen $(1986)^{9}$ and high variations in ranks and grades were observed in group 2 thereby stating that ranking and grading makes the interpretation of results easier.

In the present study, the above results emphasize that the selection of microscopic requirements, slide quality, relevant areas and relevant mitotic figures are of great help in determining the accurate mitotic counting which is of great help in estimating the biological behavior and prognosis of the patient. However, the interpretations are based on a small sample and observer size. M itotic counting has been criticized for two reasons: Lack of reproducibility of counting and the time required for an accurate assessment. The lack of reproducibility of counting is to a large extent due to the absence of strict counting protocols. In the present study, the counting was done using strict protocol and the results were highly reproducible. The criticism that mitotic counting requires extra time is unfounded and the procedure is certainly justified considering that mitotic figure count has a strong prognostic value and is a significant parameter in the selection of treatment for a patient.

In conclusion, this study shows good agreement between the investigators following a given protocol for counting the mitotic figures. As this morphological parameter is of utmost importance in diagnosis, prognosis and treatment decision making strict protocol for counting of mitotic figures is preferable.

\section{REFERENCES}

1. Baak JP. M itosis counting in tumors. Hum Pathol 1990 Jul;21(7);683-85.

2. Quinn CM, W right NA. The clinical assessment of proliferation and growth in human tumors: Evaluation of methods and applications as prognostic variables. J Pathol 1990 Feb;160(2): 93-102.

3. Thunnissen FB, A mbergen A W, K oss M, Travis W D, O' L eary TJ, Ellis IO. M itotic counting in surgical pathology: Sampling bias, heterogeneity and statistical uncertainty. Histopathology $2001 \mathrm{Jul} ; 39(1): 1-8$.

4. van Diest PJ, et al. Reproducibility of mitosis counting in 2649 breast cancer specimens: Results from the multicenter morphometric mammary carcinoma project. Human Pathol 1992 J un;23(6):603-07.

5. W oolsey JT. M easuring cell proliferation. A rch Pathol L ab M ed 1991 J un;115(6):555-57.

6. Ellison DJ, et al. M itotic counts in follicular lymphomas. Human Pathol $1987 \mathrm{M}$ ay;18(5):502-05.

7. Silverberg SG. Reproducibility of the mitosis count in the histological diagnosis of smooth muscle tumors of the uterus. Human Pathol 1976 J ul; 7(4):451-54.

8. Stenkvist $B$, et al. A nalysis of reproducibility of subjective grading systems for breast carcinoma. J Clin Pathol 1979 0ct; 32(10):979-85.

9. Donhuijsen K. M itosis counts: Reproducibility and significance in grading of malignancy. Human Pathol 1986 Nov;17(11): 1122-25.

\section{ABOUT THE AUTHORS}

\section{K Shailaja Yadav}

Professor and Head, Department of Oral and M axillofacial Surgery L enora Dental College, Rajahmundry, A ndhra Pradesh, India 


\section{Sudhir Gonuguntla}

Reader, Department of Oral Pathology, MNR Dental College, Hyderabad, A ndhra Pradesh, India

\section{Kranti Kiran Reddy Ealla (Corresponding Author)}

Senior L ecturer, Department of O ral Pathology, M NR D ental College Hyderabad, A ndhra Pradesh, India, e-mail: drekkr@yahoo.co.in

\section{Surekha Reddy Velidandla}

Senior L ecturer, Department of O ral Pathology, M NR D ental College Hyderabad, A ndhra Pradesh, India

\section{CR Charan Reddy}

Reader, Department of Oral Pathology, MNR Dental College Hyderabad, A ndhra Pradesh, India

\section{Prasanna}

Professor and Head, Department of Oral Pathology, MNR Dental College, Hyderabad, A ndhra Pradesh, India

\section{Sanjay Reddy Bommu}

Professor, Department of Oral Pathology, MNR Dental College Hyderabad, A ndhra Pradesh, India 